\title{
Preferences to Patient-Centeredness in Pre-Service Speech and Hearing Sciences Students: A Cross-Sectional Study
}

\author{
Ashley L. Dockens ${ }^{1}$, Monica L. Bellon-Harn ${ }^{1}$, and Vinaya Manchaiah ${ }^{1,2,3}$ \\ ${ }^{1}$ Department of Speech and Hearing Sciences, Lamar University, Beaumont, TX, USA, \\ ${ }^{2}$ The Swedish Institute for Disability Research, Department of Behavioural Sciences and Learning, Linköping University, Linköping, \\ Sweden, \\ ${ }^{3}$ Audiology India, Mysore, Karnataka, India
}

Received January 21, 2016

Revised April 4, 2016

Accepted May 4, 2016

Address for correspondence

Monica L. Bellon-Harn, PhD, CCC-

SLP

Department of Speech and

Hearing Sciences, Lamar University,

Beaumont, TX 77710, USA

Tel $+1-409-880-8174$

Fax $+1-409-880-2265$

E-mail monica.harn@lamar.edu
Background and Objectives: Patient-centeredness is a critical approach to rehabilitation in allied health professions and is an imperative component of evidence-based practice. However, it is unknown if patient-centeredness is valued by students enrolled in allied health. This study was aimed at determining preferences to patient-centeredness in pre-service speech and hearing students in the field of speech and hearing sciences. Subjects and Methods Pre-service speech and hearing students $(n=93)$ completed the Patient-Practitioner Orientation Scale (PPOS) and were grouped according to low, medium, or high exposure to curriculum content. Results: Across exposure levels, students exhibited high preference to patient centeredness with a mean PPOS score of 4.13 (standard deviation-0.5). A pairedsample t-test revealed a significant difference $(p \leq 0.0001)$ between the caring and sharing subscales of the PPOS with lower mean scores on the caring subscale. No significant differences were noted across levels of exposure for sharing subscale, caring subscale, or PPOS full-scale. Conclusions: Results suggest a need for curriculum enhancement focused on improved caring and empathy.

J Audiol Otol 2016;20(2):73-79

KEY WORDS: Patient-centeredness · Patient-centered care · Audiology · Speech language pathology Speech and hearing science $\cdot$ Hearing healthcare $\cdot$ Education.

\section{Introduction}

A patient-centered approach is defined as providing care that is respectful of and responsive to an individual's preference, needs, and values, and ensuring that patient values guide clinical decisions [1]. Patient-centered care in rehabilitation is fostered when patient comfort, motivation, readiness, acknowledgement and understanding of the patient as an individual, provision of useful and actionable information, and shared decision-making are included [2]. Patientcentered approaches are critically important to the rehabilitative process, especially since success is determined by patient involvement, who are unlikely to participate in intervention if it does not address individual needs [3].

This is an Open Access article distributed under the terms of the Creative Commons Attribution Non-Commercial License (http://creativecommons.org/licenses/by-nc/3.0/) which permits unrestricted non-commercial use, distribution, and reproduction in any medium, provided the original work is properly cited.
A patient's unique values and circumstances are recognized components of evidence-based practice [4]. Evidencebased practice training is essential to speech and hearing professions; thus, patient-centeredness learning modules are an imperative component of curriculum. Following the example of medical education [5], allied health fields must evaluate preference to patient-centeredness among students in order to provide the best preparatory learning experience. An understanding of current patient-centered preferences among pre-service students will contribute to development of early learning modules that promote growth across studies to become speech and hearing professionals. For this reason, the degree to which pre-service speech and hearing students value components of a patient-centered approach was evaluated. As noted, patient-centeredness in medical students has been assessed [5], but no studies have examined pre-service speech and hearing students. 


\section{Patient-centered approaches}

Patient-centeredness has been linked to positive patient outcomes (e.g., increased satisfaction, improved health outcomes, and greater quality of service delivery perception [6]) in many fields. Both medical and allied health models of patient-centered approaches have been developed with longterm rehabilitative care being a larger component of allied health models [7]. Individualization is key to allied health patient-centered approaches, and while there is no universally accepted model, most achieve this via the following components: 1) biopsychosocial perspective; 2) patient as a person; 3) shared knowledge and power; 4) therapeutic alliance; and 5) clinician as person [8]. Together these components lead to patient-centered treatment. The biopsychosocial perspective conceptualizes disorders on a number of hierarchical levels that interact in biological, psychological, and social areas [9]. The view of the patient as a person considers the patient's psychological perspective and holistically rather than diagnostically defines a patient [10]. Furthermore, shared knowledge and power between the clinician and the patient, encourages greater patient accountability for their health [11]. Therapeutic alliance similarly suggests a relationship between clinician and patient that includes shared perception, agreement on goals, and emotional context [8]. Lastly, the view of the clinician as a person considers the impact of personal factors (e.g., emotional and interpersonal responses) of the clinician to build patient-clinician relationships [12].

Preferences toward patient-centeredness are emerging as a fundamental part of the successful management of individuals with communication disorders. In the profession of audiology, researchers posited a paradigm shift from a focus hearing healthcare technologies to a patient-centered approach to rehabilitation [13]. In the profession of speech-language pathology, Duchan [14] argued that emphases on patient life participation, involvement in clinical decision-making, and cultural sensitivity are critical to the therapeutic process. She proposed that the patient's progression toward life goals be the standard to evaluate the whole course of therapy. While patient-centered approaches are a key principle of care, Ratner [15] suggested that speech-language pathologists and audiologists clinically understand the disorder, but are further behind in understanding our patients.

In an effort to increase application of patient-centered approaches, several studies have examined audiologist preference toward patient-centeredness [16-18]. Laplante-Lévesque, et al. [17] reported that audiologists have high preference to patient-centeredness. In addition, they reported that a positive association between age and years of practice with stronger preferences for patient-centeredness for audiologists in Australia [17]. Some differences were also found based on employment characteristics. For example, audiologists who worked in community education, industrial audiology, or teaching had a significantly greater preference for patientcenteredness than their peers. In contrast, audiologists who practiced in a private environment and who worked in the area of assessment of adults had a significantly lesser preference for patient-centeredness than their peers. Additionally, cultural background of the audiologist has been found to be a significant factor in the valuation of patient-centered approaches [18]. Since Australia and the United States have similar cultures and economies, it might be expected that audiologists in both countries would have comparable patientcentered preferences. However, we are not aware of any published data about audiologists' preference to patient-centeredness in the USA.

\section{Patient-centered approach and pre-service speech and hearing sciences students}

Current evidence provides possible components of instructional programs for patient-centeredness during professional training [19]. Laplante-Lévesque, et al. [17] concluded that young professionals may benefit from exposure to the work of older and more experienced professionals. Manchaiah, et al. [18] suggested that global online audiology training programs should consider cultural differences related to patientcentered preferences in their curriculum development.

Pre-service speech and hearing sciences students should be trained to examine unchangeable personal factors such as ethnic background, gender, and previous experiences and adapt services accordingly [20]. Additionally, pre-service speech and hearing students should be educated to consider changeable factors such as facilitating self-efficacy and self-advocacy to help patients cope with their condition. Moreover, it has been suggested that the first step in enhancing curriculum is identifying learner goals and objectives across courses within the curriculum [21]. However, we must first understand the preference of patient-centeredness in pre-service speech and hearing students, in order to identify related learner goals and objectives.

Previous research investigating patient-centeredness with medical students provides some direction. In particular, Wahlqvist, et al. [5] used the Patient-Practitioner Orientation Scale (PPOS) to examine gender differences in patient-centered preferences. Results indicated that female students displayed higher patient-centered preferences than male students [21]. Further, researchers examined changes in patientcentered preferences across the curriculum. Results indicated that though small, increases in student patient-centeredness occurred across the curriculum. The authors suggest that data 
regarding patient-centered preferences in pre-service students may inform the development and assessment of patient-centered objectives and evaluation of objectives.

For this reason, the aim of the present study was to understand the preference to patient-centeredness among pre-service speech and hearing sciences students. The specific aims of the study included: 1) if the pre-service speech and hearing sciences students in USA had higher or lower preferences to patient-centeredness; and 2) if there were differences among students' preferences to patient-centeredness based on the level of curriculum exposure (i.e., courses taken). We also compared students' preferences to audiologists' preferences from previously published data. This comparison is relevant since no other research regarding patient-centeredness with pre-service speech and hearing students is published. An understanding of patient-centeredness patterns and its comparison to practicing professionals may inform curriculum needs and learner objectives.

\section{Subjects and Methods}

Ethical clearance was obtained from Institutional Review Board, Office of Research and Sponsored Programs, Lamar University.

\section{Study design and participants}

A cross-sectional study was employed to gather data from pre-service speech and hearing sciences students. Enrolled students were recruited from undergraduate courses at Lamar University. The researchers provided the PPOS questionnaires to instructors of courses who dispersed them in class. No additional instructions or assistance were given to stu- dents. A total of 102 undergraduate students completed and returned the questionnaires. However, after removing those with missing data in PPOS scale, data from a sample of 93 participants were analyzed. Students were grouped according to low, medium, or high exposure to curriculum content. Low exposure was defined as 1-2 introductory courses (e.g., Introduction to Communication Disorders). Medium exposure was defined as $3-5$ courses, which included major core curriculum (e.g., Speech and Hearing Sciences). High exposure was defined as 6 or more courses, which included major advanced courses (e.g., Aural Rehabilitation, Clinical Processes). Undergraduate curricula include pre-requisite and advanced courses. Pre-requisite courses serve as foundation courses for subsequent advanced courses in specific disorders and application courses. The low exposure group included students who were not enrolled in specific disorder or applied courses. For example, low exposure students may have been enrolled in a survey course of communication disorders and/ or a general phonetic transcription courses. The medium exposure group included students who were enrolled in courses specific to a disorder area such as Introduction to Audiology. The high exposure group included students who were enrolled in application courses. For example, the course in clinical processes is a senior capstone course that includes principles of applied clinic and evidence-based practice. Table 1 provides the demographic details of the study sample.

\section{Questionnaire}

The PPOS was originally developed by Krupat, et al. [22] to study physician preferences towards patient-centeredness. The modified version of the PPOS that has been adapted to audiologists was used in this study [17]. This scale has eigh-

Table 1. Participant demographics

\begin{tabular}{|c|c|c|c|c|}
\hline \multirow{2}{*}{ Category } & \multicolumn{4}{|c|}{ Groups } \\
\hline & All groups & Low exposure & Medium exposure & High exposure \\
\hline Participant, n (\%) & $93(100)$ & $33(35.5)$ & $39(41.9)$ & $21(22.6)$ \\
\hline \multicolumn{5}{|l|}{ Gender, n (\%) } \\
\hline Female & $75(80.6)$ & $26(78.8)$ & $32(82.1)$ & $17(81.0)$ \\
\hline Male & $6(6.5)$ & $3(9.1)$ & $2(5.1)$ & $1(4.8)$ \\
\hline Not known & $12(11.8)$ & $4(12.1)$ & $5(12.8)$ & $3(14.3)$ \\
\hline Age in years (mean $\pm S D)(n=84$, data missing in 8$)$ & $22.6 \pm 5.4$ & $23.5 \pm 7.5$ & $22.3 \pm 4.3$ & $21.7 \pm 2.8$ \\
\hline \multicolumn{5}{|l|}{ Religion, n (\%) } \\
\hline Christian & $87(93.5)$ & $30(90.9)$ & $38(97.4)$ & $19(90.5)$ \\
\hline Non-religious & $6(6.5)$ & $3(9.1)$ & $1(2.6)$ & $2(9.5)$ \\
\hline \multicolumn{5}{|l|}{ Country of origin, n (\%) } \\
\hline USA & $79(84.9)$ & $27(81.8)$ & $36(92.3)$ & $16(76.2)$ \\
\hline Outside USA & $14(15.1)$ & $6(18.2)$ & $3(7.7)$ & $5(23.8)$ \\
\hline Number of core classes taken & & 2 or below & 3 to 6 & Over 6 \\
\hline
\end{tabular}


teen questions, which are scored on a 6-point Likert scale ( $1=$ strongly agree; $6=$ strongly disagree). The total score can range from 18 (most audiologist-centered) to 108 (most patient-centered). This scale has two sub-scales with 9-items each. The sharing subscale reflects the extent to which the respondent believes that patients desire information and should be part of the decision making process (e.g., patients should be treated as if they were partners with the audiologists, equal in power and status). The caring subscale reflects the extent to which the respondent sees the patient's expectations, feelings, and life circumstances as critical elements in the treatment process (e.g., a treatment plan cannot succeed

Table 2. Patient-Practitioner Orientation Scale (PPOS) mean and standard deviation (SD)

\begin{tabular}{|c|c|c|c|c|}
\hline \multirow{2}{*}{ PPOS Items } & \multicolumn{4}{|c|}{ Mean \pm SD } \\
\hline & All groups & Low exposure & Medium exposure & High exposure \\
\hline $\begin{array}{l}\text { 1. The audiologist is the one who should decide what gets } \\
\text { talked about during an appointment. }\end{array}$ & $3.44 \pm 1.4$ & $3.54 \pm 1.4$ & $3.25 \pm 1.2$ & $3.62 \pm 1.6$ \\
\hline $\begin{array}{l}\text { 2. Although health care is less personal these days, this is a } \\
\text { small price to pay for audiological advances. }\end{array}$ & $3.65 \pm 1.3$ & $3.78 \pm 1.1$ & $3.49 \pm 1.3$ & $3.76 \pm 1.4$ \\
\hline $\begin{array}{l}\text { 3. The most important part of the standard audiological } \\
\text { appointment is the hearing test. }\end{array}$ & $3.79 \pm 1.2$ & $3.76 \pm 1.3$ & $3.59 \pm 1.1$ & $4.24 \pm 1.0$ \\
\hline $\begin{array}{l}\text { 4. It is often best for clients if they do not have the full } \\
\text { explanation of their audiological condition. }\end{array}$ & $5.20 \pm 1.0$ & $5.15 \pm 1.2$ & $5.15 \pm 1.0$ & $5.38 \pm 1.0$ \\
\hline $\begin{array}{l}\text { 5. Clients should rely on their audiologists' knowledge and } \\
\text { not try to find out about their conditions on their own. }\end{array}$ & $4.16 \pm 1.4$ & $4.15 \pm 1.5$ & $4.25 \pm 1.3$ & $4.00 \pm 1.4$ \\
\hline $\begin{array}{l}\text { 6. When audiologists ask a lot of questions about a client's } \\
\text { background, they are prying too much into personal } \\
\text { matters. }\end{array}$ & $5.13 \pm 1.0$ & $5.03 \pm 1.0$ & $5.07 \pm 0.9$ & $5.38 \pm 1.0$ \\
\hline $\begin{array}{l}\text { 7. If audiologists are truly good at diagnosis and treatment, } \\
\text { the way they relate to clients is not that important. }\end{array}$ & $5.16 \pm 1.0$ & $5.00 \pm 1.2$ & $5.18 \pm 0.9$ & $5.38 \pm 1.0$ \\
\hline $\begin{array}{l}\text { 8. Many clients continue asking questions even though they } \\
\text { are not learning anything new. }\end{array}$ & $3.83 \pm 1.8$ & $3.79 \pm 1.2$ & $3.66 \pm 1.0$ & $4.19 \pm 0.9$ \\
\hline $\begin{array}{l}\text { 9. Clients should be treated as if they were partners with the } \\
\text { audiologist, equal in power and status.* }\end{array}$ & $4.23 \pm 1.2$ & $4.12 \pm 1.3$ & $4.30 \pm 1.1$ & $4.24 \pm 1.3$ \\
\hline $\begin{array}{l}\text { 10. Clients generally want reassurance rather than } \\
\text { information about their audiological condition. }\end{array}$ & $3.35 \pm 1.3$ & $3.36 \pm 1.0$ & $3.41 \pm 1.1$ & $3.24 \pm 1.2$ \\
\hline $\begin{array}{l}\text { 11. When clients disagree with their audiologist, this is a sign } \\
\text { that the audiologist does not have the client's respect } \\
\text { and trust. }\end{array}$ & $4.67 \pm 1.0$ & $4.57 \pm 1.1$ & $4.72 \pm 1.0$ & $4.76 \pm 0.9$ \\
\hline $\begin{array}{l}\text { 12. When clients disagree with their audiologist, this is a sign } \\
\text { that the audiologist does not have the client's respect } \\
\text { and trust. }\end{array}$ & $3.99 \pm 1.1$ & $4.06 \pm 1.1$ & $3.92 \pm 1.2$ & $4.00 \pm 1.0$ \\
\hline $\begin{array}{l}\text { 13. A management plan cannot succeed if it is in conflict } \\
\text { with a client's lifestyle or values.* }\end{array}$ & $4.23 \pm 1.4$ & $4.12 \pm 1.4$ & $4.20 \pm 1.4$ & $4.43 \pm 1.2$ \\
\hline $\begin{array}{l}\text { 14. Most clients want to get in and out of the audiologist's } \\
\text { office as quickly as possible. }\end{array}$ & $3.35 \pm 1.4$ & $3.18 \pm 1.4$ & $3.56 \pm 1.5$ & $3.24 \pm 1.4$ \\
\hline $\begin{array}{l}\text { 15. The client must always be aware that the audiologist is in } \\
\text { charge. }\end{array}$ & $3.44 \pm 1.0$ & $3.57 \pm 1.0$ & $3.30 \pm 1.0$ & $3.48 \pm 1.0$ \\
\hline $\begin{array}{l}\text { 16. It is not that important to know a client's culture and } \\
\text { background in order to treat the client's audiological } \\
\text { condition. }\end{array}$ & $5.20 \pm 1.3$ & $5.09 \pm 1.4$ & $5.23 \pm 1.2$ & $5.33 \pm 1.1$ \\
\hline $\begin{array}{l}\text { 17. Humour is a major ingredient in the audiologist's } \\
\text { management of the client.* }\end{array}$ & $4.05 \pm 1.2$ & $4.00 \pm 1.1$ & $4.10 \pm 1.1$ & $4.05 \pm 1.0$ \\
\hline $\begin{array}{l}\text { 18. When clients look up audiological information on their } \\
\text { own, this usually confuses more than it helps. }\end{array}$ & $3.51 \pm 1.1$ & $3.63 \pm 1.0$ & $3.36 \pm 1.1$ & $3.62 \pm 1.2$ \\
\hline Sharing subscale & $4.29 \pm 0.6$ & $4.20 \pm 0.6$ & $4.21 \pm 0.5$ & $4.46 \pm 0.7$ \\
\hline Caring subscale & $3.97 \pm 0.4$ & $3.95 \pm 0.4$ & $3.98 \pm 0.4$ & $4.01 \pm 0.4$ \\
\hline PPOS full-scale & $4.13 \pm 0.5$ & $4.10 \pm 0.4$ & $4.10 \pm 0.5$ & $4.24 \pm 0.5$ \\
\hline
\end{tabular}

Score of 1 (strongly agree)=most clinician-centered; Score of 6 (strongly disagree)=most patient-centered. Items 9, 13, and 17 (*) are reversely worded items which were reverse scored 
if it is in conflict with a patient's lifestyle or values). This scale found to have good factor structure and acceptable internal consistency $(\alpha=0.78)$.

\section{Data analysis}

In the first instance, descriptive statistics (i.e., mean, standard deviation) were performed. No violations were found in normality and homogeneity assumptions. T-test was used to compare means of current study results with previously published results and also compare the mean difference between subscales. In addition, a one-way analysis of variance (ANOVA) was used to compare the differences in mean PPOS scores for different groups of pre-service speech and hearing students based on the level of exposure. An alpha level of 0.05 was used to determine significance.

\section{Results}

Table 2 presents the PPOS mean scores and standard deviations. The pre-service speech and hearing sciences students had a mean PPOS score of 4.13 (standard deviation-0.5), which we compared with Australian audiologists (i.e., 4.46) using a one-sample t-test. The results showed that pre-service speech and hearing students had significantly lower scores when compared to the audiologists [ $\mathrm{t}(92)=-6.804), p \leq 0.0001]$.

We used paired-sample t-test to compare the mean scores of sharing subscale and caring subscale of pre-service speech and hearing students. Results suggested that the students had significantly lower mean scores in caring subscale when compared to sharing subscale $[\mathrm{t}(92)=5.53), p \leq 0.0001]$. In addition, the PPOS mean scores from different undergraduate groups based on exposure levels were analyzed using a one way between-subjects ANOVA. No significant differences were found for sharing subscale $[\mathrm{F}(2,90)=1.185, p=0.310]$, caring subscale $[\mathrm{F}(2,90)=0.111, p=0.895]$ and the PPOS fullscale $[\mathrm{F}(2,90)=0.718, p=0.490]$. Fig. 1 shows the mean and $95 \%$ confidence intervals of mean for PPOS full-scale among three groups of undergraduate speech and hearing sciences students.

\section{Discussion}

The current study was aimed at understanding the preference to patient-centeredness in pre-service speech and hearing sciences students. Results indicate a high preference to patient-centeredness in pre-service speech and hearing sciences students in USA. However, no difference among exposure groups was noted.

The mean PPOS scores in this study (4.13) is comparable

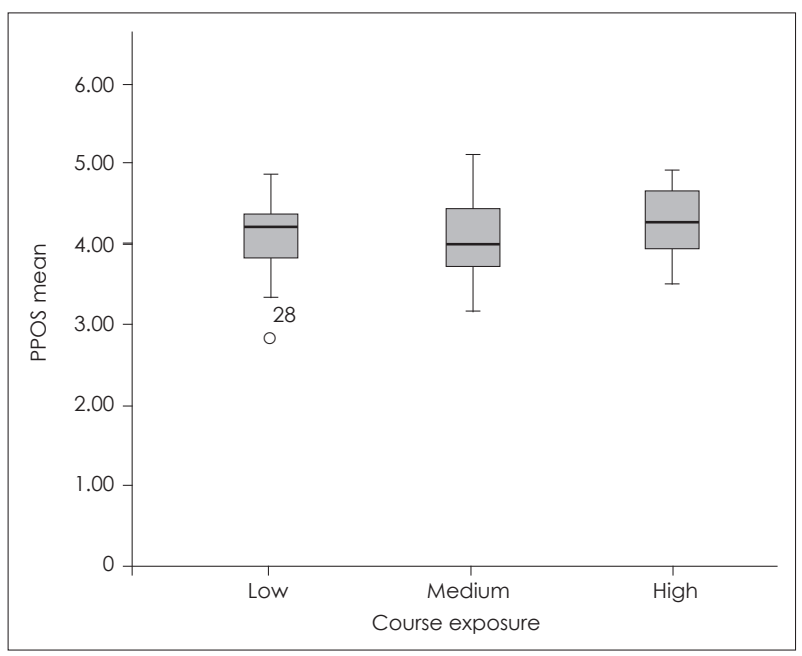

Fig. 1. PPOS: Patient-Practitioner Orientation Scale.

to mean pre-service medical students' scores reported in Wahlqvist, et al. [5], which were 4.20 and 4.36 for men and women respectively. Wahlqvist, et al. [5] reported no decline of patient-centered attitudes across the curriculum, which is consistent with this study. This suggests that established values are maintained and supported as undergraduate students progress in years. Wahlqvist, et al. [5] identified a significant increase in PPOS mean scores, albeit small, across the curriculum. In this study scores did increase, though no significant differences were noted. Scores in this study are comparable to means scores of practicing audiologist in Australia (4.46) [17]. Overall, a high preference to patient centeredness is indicated.

Of the scores that contributed most to the overall caring subscale score, item 16 (i.e., it is not that important to know a patient's culture and background in order to treat their audiological condition) received the highest preference for patient-centeredness. The mean score of 5.20 indicates a strong preference to understanding the patient's culture. Of the scores that contributed most to the overall sharing subtest score, item 4 (i.e., it is often best for patients if they do not have the full explanation of their audiological condition) indicated students' preference for ensuring patients fully understand their condition. Students indicated less patient-centeredness than on item 10 (i.e., patients generally want reassurance rather than information about their audiological condition) and 14 (i.e., most patients want to get in and out of the audiologist's office as quickly as possible) than other items. Both of these items are within the caring subtest score.

Although students had a slightly lower score than audiologists in Australia, some scores on specific items were comparable (i.e., item 4, 6, and 7). On the other hand, students indicated less patient-centeredness than audiologists on item 
2 (i.e., although health care is less personal these days, this is a small price to pay for audiological advances). The mean score of 5.20 on item 16 indicates a stronger preference to understanding the patient's culture than found in LaplanteLévesque, et al. [17] who reported a mean of 4.95. Pre-service students indicated less patient-centeredness than audiologists on item 14. While these comparisons are interesting, it is important to note that although Australia and United States have similar culture and economies, it may be insufficient to compare the data from two countries. While the resulting differences may to some degree be attributed to level of training between professionals and pre-service students, other factors may have influence. Greene et al. [23] suggest that patientcentered care and patient experiences can be collectively influenced by multiple factors including clinical, structural, and interpersonal attributes. Patient-centered preference could also differ between the two countries based on individuals' education (e.g., curricula) and ethics, as well as personality [24]. For this reason, the comparisons of these results are exploratory and may not be generalizable.

No significant differences among different exposure levels may be explained in several ways. First, it may be that students who are attracted to human service professions such as speech and hearing are more likely to be patient-centered prior to course initiation. Second, it may be that students enroll in related professional electives as components of their degree plan, which include patient-centeredness concepts and values prior to taking speech and hearing professional courses. Third, the initial course in the speech and hearing curriculum (i.e., Introduction to Communication Disorders) included discussions on evidence-based practice across scope of practice. One key component is patient and family values. Finally, there is overlap of course type within the groups and no clear demarcation about course content. For example, students in the medium-exposure group may have taken a core course in which content included disorder type and management principles.

A difference between scores on the sharing and caring subscales was noted. It may be that the caring aspect relates to empathy, which may further develop over time with experience. As students participate in the patient-clinician relationship, they may acquire a deeper patient perspective to the clinical process. Typically, active participation in the clinical process does not occur until graduate training.

\section{Strengths and limitations}

This study is the first exploration of preference to patientcenteredness among speech and hearing sciences students of which we are aware. One notable limitation was the catego- rization of low, medium, and high exposure. The students investigated in this proposal did not matriculate through the years in a lockstep manner. As such, students in the medium exposure group may have taken courses that included approaches to patient care. Further, students in the low exposure group completed or were enrolled in foundation courses (e.g., Introduction to communication science and disorders). These courses emphasize evidence-based practice, which includes focus on patient and family needs. It should be noted that since undergraduate programs vary across U.S. universities, the results must be viewed with caution.

\section{Study implications and further research}

Findings of high preference to patient-centeredness among students in this study were positive and promising. However, when comparing these students to practicing audiologists, there was no published data on preference to patient-centeredness among audiologists in USA. Hence, we used data from Australian audiologists to compare preferences to patient-centredness with pre-service students in USA, which highlights the need for future studies to develop country specific data. Additionally, a difference between subscales of sharing and caring was found with the caring subscale not as positive as the sharing subscale. It is suggested that patientcentered caring is related to empathy that develops over experience and time. Researchers have exhibited that empathy is a major contributor to patient-clinician trust, which is core to patient-centeredness. Preminger and colleagues suggest that patient trust is strengthened with empathy, good communication skills, shared-decision making, self-management promotion for the patient, and technical competence of the clinician [25]. Since direct experience is not as common in undergraduate education as it is graduate education, the potential for experiential growth in empathy is limited. Findings of this study suggest a need for inclusion of coursework and experience that support development of empathy. Curriculum changes could be then evaluated for growth of student empathy via the PPOS caring subscale.

Since pre-service speech and hearing training programs vary, questions remain whether or not patient-centered values are comparable across universities. No data has been previously published data exists from PPOS in pre-service speech and hearing students from which to compare the mean scores. This study may serve as a basis from which to compare other student samples. Additionally, many programs include a post-baccalaureate curriculum. Do similar patterns of patient-centered values emerge? While studies have been conducted to examine audiologist, questions remain whether or not difference among preferences in audiologists and 
speech-language pathologists exist. Additionally, factors of pre-work experiences in related fields, motivation for studying speech and hearing sciences, and family history related to the field were not evaluated, which may influence preferences to patient-centeredness. Further research should consider these factors. Finally, since patient-centeredness is linked to health outcomes and evidence-based questions, future studies should investigate relationships between preference to patient-centeredness and observable clinical behavior.

\section{Conclusions}

Results of the present study suggest that undergraduate students may have strong inclination toward patient-centeredness. The undergraduate students in this study did not vary across levels of exposure to curriculum content. High levels of preference to patient-centeredness were noted whether or not the students were in low, medium, or high exposure. However, their preference in the sharing subscale was higher than preference in the caring subscale. Generally, these results suggest that undergraduate students reported high preference to patient-centeredness.

\section{Conflicts of interest}

The authors have no financial conflicts of interest.

\section{REFERENCES}

1) Institute of Medicine (IOM), an arm of the National Academy of Sciences. Crossing the Quality Chasm: A New Health System for the 21st Century. Washington, DC: National Academy Press;2001.

2) Poost-Foroosh L, Jennings MB, Cheesman MF. Comparisons of client and clinician views of the importance of factors in client-clinician interaction in hearing aid purchase decisions. J Am Acad Audiol 2015;26:247-59.

3) Cott CA. Client-centred rehabilitation: client perspectives. Disabil Rehabil 2004;26:1411-22.

4) Dollaghan C. The Handbook for Evidence-Based Practice in Communication Disorders. Baltimore, MD: Paul Brooks Publishing Co.; 2007.

5) Wahlqvist M, Gunnarsson RK, Dahlgren G, Nordgren S. Patientcentred attitudes among medical students: gender and work experience in health care make a difference. Med Teach 2010;32:e191-8.

6) Stone S. A retrospective evaluation of the impact of the Planetree patient-centered model of care on inpatient quality outcomes. HERD 2008;1:55-69.
7) Grenness C, Hickson L, Laplante-Lévesque A, Davidson B. Patientcentred care: a review for rehabilitative audiologists. Int J Audiol 2014;53 Suppl 1:S60-7.

8) Mead N, Bower P. Patient-centredness: a conceptual framework and review of the empirical literature. Soc Sci Med 2000;51:1087-110.

9) Engel GL. The clinical application of the biopsychosocial model. J Med Philos 1981;6:101-23.

10) Bower P. Understanding patients: implicit personality theory and the general practitioner. Br J Med Psychol 1998;71(Pt 2):153-63.

11) Grol R, de Maeseneer J, Whitfield M, Mokkink H. Disease-centred versus patient-centred attitudes: comparison of general practitioners in Belgium, Britain and The Netherlands. Fam Pract 1990;7:100-3.

12) Winefield HR, Murrell TG, Clifford JV, Farmer EA. The usefulness of distinguishing different types of general practice consultation, or are needed skills always the same? Fam Pract 1995;12:402-7.

13) Danermark B. Patient-centred audiological rehabilitation: facilitating and hindering factors for implementation. ENT \& Audiology News 2014;23:71-2.

14) Duchan JF. Impairment and social views of speech-language pathology: clinical practices re-examined. Int J Speech Lang Pathol 2001; 3:37-45.

15) Ratner NB. Evidence-based practice: an examination of its ramifications for the practice of speech-language pathology. Lang Speech Hear Serv Sch 2006;37:257-67.

16) Grenness C, Hickson L, Laplante-Lévesque A, Davidson B. Patientcentred audiological rehabilitation: perspectives of older adults who own hearing aids. Int J Audiol 2014;53 Suppl 1:S68-75.

17) Laplante-Lévesque A, Hickson L, Grenness C. An Australian survey of audiologists' preferences for patient-centredness. Int J Audiol 2014;53 Suppl 1:S76-82.

18) Manchaiah V, Gomersall PA, Tomé D, Ahmadi T, Krishna R. Audiologists' preferences for patient-centredness: a cross-sectional questionnaire study of cross-cultural differences and similarities among professionals in Portugal, India and Iran. BMJ Open 2014;4:e005915. Stewart M, Brown JB, Weston WW, McWhinney IR, McWilliam CL, Freeman TR. Patient-Centered Medicine-Transforming the Clinical Method. 2nd ed. London: Radcliffe Medical Press;2003.

19) Howe TJ. The ICF Contextual Factors related to speech-language pathology. Int J Speech Lang Pathol 2008;10:27-37.

20) Peters KA. Including service learning in the undergraduate communication sciences and disorders curriculum: benefits, challenges, and strategies for success. Am J Audiol 2011;20:S181-96.

21) Krupat E, Putnam SM, Yeager C. The fit between doctors and patients: can it be measured? J Gen Intern Med 1996;11:134.

22) Greene SM, Tuzzio L, Cherkin D. A framework for making patientcentered care front and center. Perm J 2012;16:49-53.

23) Sine DM, Sharpe VA. Ethics, risk, and patient-centered care: how collaboration between clinical ethicists and risk management leads to respectful patient care. J Healthc Risk Manag 2011;31:32-7.

24) Preminger JE, Oxenbøll M, Barnett MB, Jensen LD, LaplanteLévesque A. Perceptions of adults with hearing impairment regarding the promotion of trust in hearing healthcare service delivery. Int J Audiol 2015;54:20-8. 